\title{
A Simulation Study of Common Mobility Models for Opportunistic Networks
}

\author{
Muhammad Abdulla and Robert Simon \\ Department of Computer Science \\ George Mason University \\ Fairfax, VA 22030 \\ Email: \{mabdulla, simon\}@cs.gmu.edu
}

\begin{abstract}
Understanding mobility characteristics is important for the design and analysis of routing schemes for mobile ad hoc networks (MANETs). This is especially true for mobile opportunistic networks where node mobility is utilized to achieve message delivery. In this paper, we study the properties of common mobility models. Specifically, we study inter-contact times of mobile nodes in Random Waypoint and Random Direction mobility models under opportunistic network settings. We also introduce a modified Random Waypoint model with hot-spots to study its mobility properties. Through extensive simulation study, we provide simulation results for mobility properties of Random Waypoint and Random Direction models. Further, our modified Random Waypoint with hot-spots model is also found to show an approximate power-law and exponential inter-contact time dichotomy found in real-world mobility traces as described in recent literature.
\end{abstract}

\section{Introduction}

Understanding mobility characteristics is important for the design and analysis of routing schemes for mobile ad hoc networks. This is especially true for mobile opportunistic networks where node mobility is utilized to achieve message delivery. Routing schemes for traditional mobile ad hoc networks (MANETs) assume that nodes are well connected most of the time. Generally, proactive schemes, where nodes try to keep up to date routing information [18], or reactive schemes, where nodes find routing paths on demand [19], are used to achieve message delivery. Both schemes assume that there exists an end-to-end path from source to destination at the time of message transfer. However, such assumptions do not hold true when the mobile network is sparse and is intermittently connected. In such systems network partitions and large delays are common. Under these conditions, traditional MANET routing algo- rithms fail to work well, as proactive schemes do not converge, while reactive schemes fail to find a path to the destination.

Routing methods for such sparse mobile networks use a different paradigm for message delivery; these schemes utilize node mobility by having nodes carry messages, waiting for an opportunity to transfer messages to the destination or the next relay rather than transmitting them over a path $[12,20,23,24,29]$. Under such opportunistic network (ON) routing protocols [25], nodes forward messages only when they encounter the appropriate relay or the destination node. Due to this dependence on mobility, understanding mobility characteristics such as inter-contact times of mobile nodes within each other or at a static location plays an important role in the design and analysis of routing algorithms under this paradigm.

Under opportunistic routing message delay is an important performance metric, not only because it is a major concern for many applications, but also because of its effect on other performance metrics, such as message delivery ratio and buffer occupancy, etc. This is especially true when there is a message expiration time associated with messages. Consequently, message delay has been the main focus of much theoretical work in this field $[11,21-24,28]$. Therefore, it is important to understand the characteristics of mobility models that contribute to message delay. Understanding such characteristics of mobility models not only helps us to relate experimental results to analytical models, but also enables us to choose appropriate mobility models in simulation studies when the mobility characteristics of real world applications are known.

In this paper, we extend analytical results given in our paper [1] through simulation studies to understand the mobility characteristics of commonly used mobility models. As discussed in previous literature [1,11], the inter-contact times in Random Waypoint and Random Direction models under opportunistic network conditions can be closely approximated as exponential distribution. In this work, we perform simulation studies for a detailed look on the inter- 
contact times in Random Waypoint and Random Direction mobility models, as well as on contributing factors such as relative speed of nodes under such mobility models that are important for the performance analysis of routing schemes. Further, we extend the Random Waypoint model with hotspots and study the mobility characteristics of this model through simulation studies.

The extension of Random Mobility model with hotspots is motivated by recent progress on the analysis of real-world mobility traces. Although exponential inter-contact times have been observed in mobility models described above, recent studies have shown that inter-contact times observed in real-world traces of mobile ad hoc networks have different characteristics. Although a power-law distribution of intercontact times is described in [7], Karagiannis et. al. show that there exist a power-law and exponential dichotomy in inter-contact times, where inter-contact times are distributed according to power-law distribution up-to a time, and exponential afterwards [14]. For this purpose, we explore the use of hot-spots under Random-Waypoint model. Through simulation studies, we have found that under certain parameters, inter-contact times approximately show such dichotomy. This model, where nodes are anchored to different hot-spots, also introduces non-uniformity of inter-contact among nodes, which can be used for scenarios where different nodes have different inter-contact rates, as studied in [13].

To analyze inter-contact times we first view node activities as interleaving encounters and departures-nodes spend some time within each other's radio range after a contact, and spend some more time before they encounter each other again. Since message transfers only occur when nodes meet each other, we focus on the node inter-contact time and its distribution. This is because the inter-contact time is the main contributing factor to message delays as the effects of the contact time and the message transfer time are comparatively small under opportunistic network conditions.

The rest of the paper is organized as follows. Section 2 goes over the related work. Section 3 discusses the stochastic mobility properties of the Random Waypoint and Random Direction mobility models. Section 4 presents experimental results. Finally, Section 5 concludes the paper.

\section{Related Work}

Mobility models play an important role in the simulation study of mobile networks. Two common mobility models are the Random Waypoint (RWP) and Random Direction (RD). Other mobility models are proposed by different groups $[6,8,9,25]$. Also, issues such as non-uniform node distribution and speed decay have been addressed for the RWP model $[3,27]$. However, RWP and RD mobility models are currently widely used in network simulations and are the focus of our study.

The stochastic properties of the Random Waypoint mobility model have been extensively studied [2,3,15,17]. Similar studies are also available for the Random Direction model $[4,5,16]$. Most of these studies focus on node distributions, epoch lengths, and movement directions, and are a foundation for further analysis of node movement characteristics under the mobility models. Analytical results for transient behavior of nodes under RWP and RD are presented in [10]. Results for the expected hitting time and meeting time in RWP and RD models are given in [25] in the context of mobility-assisted routing. Similar results are presented in [11] for the analysis of message delay under sparse networks. In our study, we focus on the rate and the distribution of inter-contact times under RWP and RD models.

The near-exponential distribution of inter-contact times in RWP and RD models surfaces in different experimental studies $[11,26]$. In [11], authors analyze the message delay in epidemic routing under the assumption that the intercontact times are exponentially distributed, and experimentally verify the validity of the assumption under Random Waypoint and Random Direction models. Based on this exponentiality assumption, further modeling of Epidemic routing performance is provided in [28]. Many studies also use Markovian model for node inter-contact times with or without assuming RWP or RD mobility model [21-23, 26]. In [1], it is shown that the inter-contact times in these mobility models can be closely approximated as exponential distribution under opportunistic network conditions. This helps to simplify the analysis of routing schemes under RWP or RD models, and to relate experimental results to analytical models. It also enables us to use these two mobility models for simulation when the inter-contact times are known to be exponentially distributed. In this paper, we examine the inter-contact times of mobile nodes through extensive simulation study. Although, in general, the exponentiality assumption holds, we also find that the intercontact time distribution under Random Waypoint and Random Direction models differ from the exponential distribution in different ways for small inter-contact times.

Real world mobility traces have also been used for the study of mobility characteristics for opportunistic networks. Results on node inter-contact times based on human mobility traces are presented in [7], providing empirical evidences that the inter-contact times are distributed according to power law up-to moderate time scale. This is in disagreement with the common exponentiality assumptions. However, recent work by Karagiannis et al [14] presented evidence showing that although the inter-contact time follows power law distribution up-to certain time, it shows exponential decay afterwards. However, as we discussed above, the two common mobility models do not show such charac- 
teristics. For this reason, we extend the Random Waypoint model by introducing hot spots, to which different nodes are anchored, with specific pause time characteristics. Under certain settings, we found that node inter-contact times have characteristics that are similar to real-world traces described in [14] - approximate power-law distribution at the beginning, and exponential at the tail.

Understanding the movement characteristics of common mobility models and performance analysis are important in the understanding and evaluation of MANET routing schemes. We believe that our analysis of mobility characteristics and performance metrics will be helpful in the analysis and design of various opportunistic routing schemes for intermittently connected mobile networks.

\section{Stochastic Properties of Mobility Models}

In this section, we study the statistical properties of node encounters, focusing on node inter-contact times and contact times using two commonly used mobility models: Random Direction and Random Waypoint. We primarily focus on the inter-contact time as it is the main component of delay in opportunistic networks. Before we continue our discussion we go over related background and our assumptions.

\subsection{Preliminaries}

In this study, we focus on two epoch-based mobility models: Random Waypoint (RWP) and Random Direction (RD). For practical purposes, we consider a twodimensional system space $\mathcal{A}$ of size $A$ as a square area of width $a$ or a circular region with radius $a$. The movement of a node from a starting position to its next destination is denoted as a movement epoch, or an epoch in short. In epoch-based mobility schemes, a node starts from point $P_{i} \in \mathcal{A}$, and moves to another point $P_{i+1} \in \mathcal{A}$ according to the movement semantics of the mobility model to complete the epoch. It then pauses for a random amount of time $T_{p}$, randomly chosen with the expected value of $\bar{T}_{\text {pause }}$. This process repeats in this manner. We use $\bar{L}$ to denote average epoch distance, and use $\bar{T}$ to denote average epoch duration. The movement speed $v$ is uniformly and randomly chosen from $\left[v_{\min }, v_{\max }\right]$, where $0<v_{\min }<v_{\max }<\infty$.

Formally, Random Waypoint can be specified as a stochastic process

$$
\left\{P_{i}, T_{p, i}, V_{i}\right\}_{i \in \mathbb{N}}=\left\{\left(P_{1}, T_{p, 1}, V_{1}\right),\left(P_{2}, T_{p, 2}, V_{2}\right), \cdots\right\}
$$

where $T_{p, i}$ is the pause time at waypoint $P_{i}$, and $V_{i}$ is the velocity of node during the $i^{\text {th }}$ epoch. $P_{i}$ is assumed to be independently and identically distributed (i.i.d) at random, uniformly chosen from $\mathcal{A}$. The Random Direction model can be specified similarly.
Nodes are assumed to have circular radio range with radius $r$. For the case of sparse mobile networks, we assume that $r \ll a$ and that $r \ll \bar{L}$.

From the viewpoint of a mobility model, node movements consist of interleaving periods of movements and pauses. From an application's point of view, node $N$ sees the movements of another node $M$ in terms of the time that $M$ spends in its radio range, which we call as contact time, and the inter-contact time between two contacts. The contact time is defined as the time elapsed from a node's entry into another node's radio range until its consequent exit. The inter-contact time is defined as the time passed since previous exit until next entry into the radio range. Below we mainly discuss inter-contact times.

\subsection{Inter-contact Times in Random Way- point Model}

It is shown in [1] that the inter-contact times under Random Waypoint model can be closely approximated as exponential when the radio range $r$ is much smaller than the $a$, for circular or square regions.

The CDF of inter-contact times of a mobile node at a static point $P(x, y)$ is approximated

$$
F(t)=1-e^{-\lambda t}
$$

where $\lambda=2 r \bar{v} f(x, y)$. Here $f(x, y)$ is PDF of node distribution at point $P(x, y)$ [2], and $\bar{v}$ represents the average movement speed of mobile node [27]:

$$
\bar{v}=\frac{\bar{L}}{\bar{T}}=\frac{v_{\max }-v_{\min }}{\ln \left(v_{\max } / v_{\min }\right)}
$$

The CDF of inter-contact times between two mobile nodes is approximated as follows:

$$
F(t)=1-e^{-\lambda t}
$$

where $\lambda=2 \rho r \tilde{v} / A$. Here, $\tilde{v}$ represents the relative speed of mobile nodes, and is given as follows [1]:

$$
\begin{aligned}
\tilde{v}= & \frac{1}{2 \pi \ln ^{2}\left(\frac{v_{\max }}{v_{\min }}\right)} \int_{v_{\min }}^{v_{\max }} \int_{v_{\min }}^{v_{\max }} \int_{0}^{2 \pi} \\
& \frac{\sqrt{\left(v_{2} \sin \theta\right)^{2}+\left(v_{1}-v_{2} \cos \theta\right)^{2}}}{v_{1} v_{2}} d \theta d v_{2} d v_{1}
\end{aligned}
$$

where $\theta$ is the relative angle between $\vec{v}_{1}$ and $\vec{v}_{2}$, measured counter-clockwise from the direction of $\vec{v}_{1}$.

As shown in [1], we take that $\theta$ is uniformly distributed.

Although $\rho$ is considered to be a constant specific to Random Waypoint model in [11], we see that the value of this factor depends on the shape of $\mathcal{A}$, and can be calculated 
analytically or numerically when $f(x, y)$ is known. For instance, for the square and the circular areas, approximate values of $\rho$ can be calculated as $1.3683[11]^{1}$ and 1.4155 (see Appendix), respectively.

We can see from (2) above that for different pairs of $v_{\text {min }}$ and $v_{\max }$ that give the same $\bar{v}$, the expected relative speed may vary. That is, knowing $\bar{v}$ is not sufficient to obtain the relative speed, $\tilde{v}$, without taking the distribution of the speed into account. Under the simplifying condition that $v_{\min }=v_{\max }=\bar{v}$ or $v_{\min } / v_{\max } \approx 1$, the relative speed can be approximated as follows:

$$
\tilde{v}=\frac{\bar{v}}{2 \pi} \int_{0}^{2 \pi} \sqrt{\sin ^{2} \theta+(1-\cos \theta)^{2}} d \theta=\frac{4 \bar{v}}{\pi}
$$

where $\theta$ is the angle between two movement directions.

\subsection{Inter-contact Times in Random Direc- tion Model}

In the Random Direction model, at the beginning of each epoch nodes randomly choose a direction uniformly from $[0,2 \pi)$, and randomly selects a speed uniformly from $\left[v_{\min }, v_{\max }\right]$. The epoch duration is exponentially generated with an average of $\bar{L} / \bar{v}$. Upon hitting the border, nodes either reflect back, or wrap-around to appear from the opposite end.

Compared to the RWP model, the node distribution in RD model is uniform: $f(x, y)=1 / A[4,16]$, and the average speed is simply given as $\bar{v}=\left(v_{\max }+v_{\min }\right) / 2$. When the epoch duration is small compared to average inter-contact time, we can follow the analysis for RWP and show that the inter-contact times are exponentially distributed, where the CDF approximation is given as

$$
F(t)=1-e^{-\lambda t}
$$

where $\lambda=2 r \bar{v} / A$.

When two nodes move according the RD model, the CDF for inter-contact time is approximated as

$$
F(t)=1-e^{-\lambda t}
$$

where $\lambda=2 r \tilde{v} / A$. Here $\tilde{v}$ is the relative speed of two nodes under RD model, which can be given as follows:

$$
\begin{aligned}
\tilde{v}= & \frac{1}{2 \pi\left(v_{\max }-v_{\min }\right)^{2}} \int_{v_{\min }}^{v_{\max }} \int_{v_{\min }}^{v_{\max }} \int_{0}^{2 \pi} \\
& \sqrt{\left(v_{2} \sin \theta\right)^{2}+\left(v_{1}-v_{2} \cos \theta\right)^{2}} d \theta d v_{2} d v_{1}
\end{aligned}
$$

where $\theta$ is the relative angle between $\vec{v}_{1}$ and $\vec{v}_{2}$, measured counter-clockwise from the direction of $\vec{v}_{1}$.

\footnotetext{
${ }^{1}$ This value is calculated in [11] based on analytical results from [15]. However, note that numerically calculating according to analytical results from [3] gives 1.3805 .
}

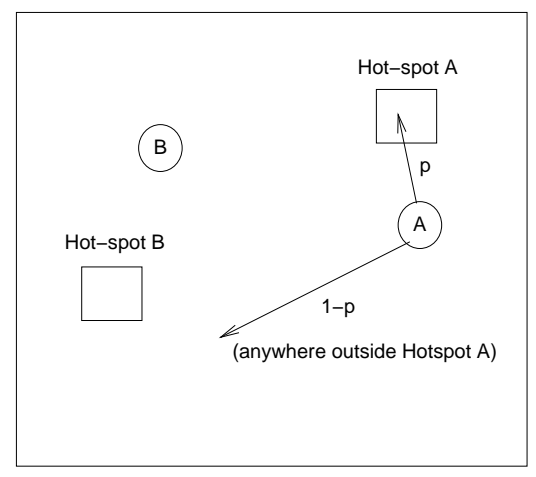

Figure 1. Random Waypoint with Hotspots

The results for $\tilde{v}$ under RD model when $v_{\max }=v_{\min }$ or $v_{\max } / v_{\min } \approx 1$ is the same with RWP model as given in (3).

Note that our result for inter-contact rate for RWP is different from the result for meeting times given in [25], where the difference of inter-contact rates among mobile nodes under RWP and RD models is attributed to relative speed on the account that the relative movement angle is not uniform under RWP model. As shown in [1], we see that the relative movement angle can also be considered uniform under RWP, and that the difference in inter-contact rates under these two models is a combined result of non-uniform node distribution under RWP as explained by factor $\rho$ and the differences in the calculation of the relative movement speed for RWP and RD models, as shown by Equations (2) and (5), respectively.

\subsection{Random Waypoint with Hotspots}

We have two motivations for extending Random Waypoint model with hot-spots. First is to see if the intercontact distributions observed in real-world mobility traces as shown in $[7,14]$ can be closely approximated using this extended model. Second is to introduce non-uniformity of inter-contact rates among different pairs of nodes, which is also closer to real-world scenarios, as discussed in [13].

In this extended model, a node may have one or more hot-spots that it may visit more often. The pause times at such hot-spots may also be different compared to areas outside of the hot-spot. Figure 1 depicts a very simple scenario where Node $A$, which is currently outside of Hot-spot A, chooses some point within Hot-spot A with a probability $p$, and any other point outside of the hot-spot with probability $1-p$. This model generalized for cases when there are more than one hotspots as follows.

Given a system area $\mathcal{A}$, assume that there are $N$ hotspots, $H_{1}, H_{2}, \cdots, H_{N}$, and a mobile node $M$. At the beginning of an epoch, node $M$ randomly selects a hotspot $H_{i}$ with probability $p_{i}$, and moves towards a point randomly 
selected within $H_{i}$. We further assume that the hotspots do not overlap. Even if hotspots overlap, we can designate overlapped regions as new hotspots and adjust the probabilities accordingly. Let $p_{0}$ denote the probability that $M$ chooses a point outside of any hotspot, then $\sum_{i=0}^{N} p_{i}=1$ should hold true, where $0 \leq p_{i}$ for $i=0, \cdots, N$.

This model can be extended to multiple nodes by assigning possibly different probabilities to different nodes for a given hotspot, while ensuring the properties for each node as discussed above. Non-uniformity of inter-contact times between different pairs of nodes can be introduced by adjusting the hotspot probabilities for different nodes. As to the power-law and exponential dichotomy of nodal intercontact times observed in real-world mobility traces, we study two nodes moving according to Random Waypoint with Hotspots model, and observe the effects of choosing hotspot locations and changing hotspot probabilities on the distribution of inter-contact times. As will be discussed in next section, we observe that such power-law and exponential dichotomy in inter-contact time distribution can be observed using our model under certain set of parameters.

\section{Experimental Results}

In this section, we present experimental results for mobility characteristics. The goal of our experiments is to obtain detailed results for inter-contact times in Random Waypoint and Random Direction. Since the relative velocity of mobile nodes is importance in the understanding of the inter-contact rates, we also study the relative velocities and relative movement angles. We also present results for our extended Random Waypoint model with hotspots, to show that the power-law and exponential dichotomy observed real-world mobility traces can be approximately produced under certain settings.

\subsection{Experimental Settings}

Most of our experiments use the $n s$-2 network simulator extended with our own code. We also use our custom simulator for experiments in circular areas, as well as for detailed measurements of the relative speed and movement angles.

The default settings for $n s$ - 2 simulations are as follows. Each simulation run has 40 nodes moving according to the specified mobility model in a $6000 \mathrm{~m} \times 6000 \mathrm{~m}$ square area. By default, nodes have a radio range of $250 \mathrm{~m}$. Minimum and maximum speeds, $v_{\min }$ and $v_{\max }$, are $3 \mathrm{~m} / \mathrm{s}$ and $10 \mathrm{~m} / \mathrm{s}$, respectively. Nodes use $H E L L O$ signals to announce their presence. The HELLO interval is set to $3 \mathrm{sec}-$ onds.

To measure contact and inter-contact times, a node stores the time when another node is found in its radio range for

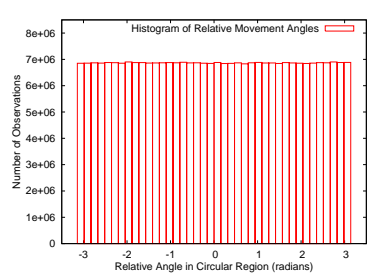

(a) Circular Region

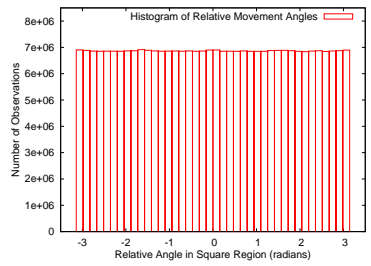

(b) Square Region

\section{Figure 2. Relative Movement Angle Distribu- tion}

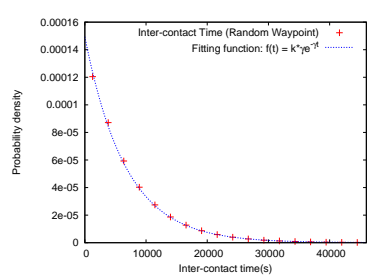

(a) Histogram of Inter-contact Times

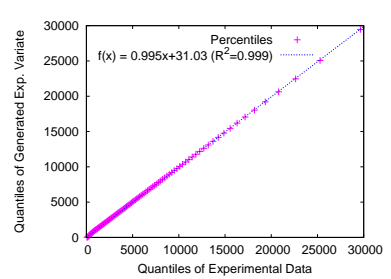

(b) Linear Regression Analysis

\section{Figure 3. Exponentiality of Inter-contact Times under Random Waypoint Model}

the first time. When a HELLO message is not heard within a HELLO interval (plus a small tolerance time), the node is marked as gone out of radio range and the contact time is recorded as the time elapsed. Inter-contact time is recorded when it receives a HELLO message again from the destination. The process repeats in this manner.

For the relative speed and relative movement angles, we use our custom simulator, and calculate the relative speed and relative movement angles of a pair of nodes at every 0.1 second. In Random Direction we use the reflection model, where nodes bounce back when they hit the borders of the region. We ran each experiment 29 times with random seeds.

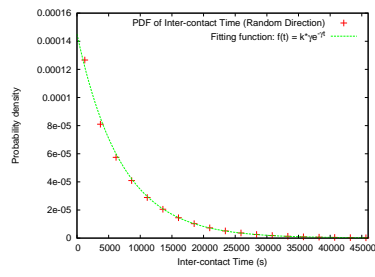

(a) Histogram of Inter-contact Times

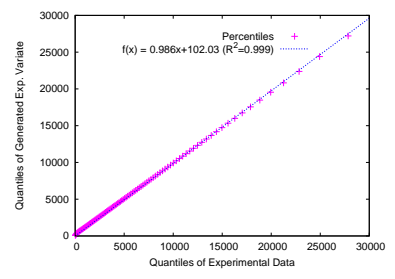

(b) Linear Regression Analysis
Figure 4. Exponentiality of Inter-contact Times under Random Direction Model 
Table 1. Relative Speed of Mobile Nodes under Random Waypoint Model

\begin{tabular}{|c|c|c||c|c||c|c|}
\hline \multicolumn{3}{|c||}{ Speed Settings } & \multicolumn{2}{c||}{ Analytical } & \multicolumn{2}{c|}{ Empirical } \\
\hline$v_{\min }$ & $v_{\max }$ & $\bar{v}$ & $\tilde{v}$ & $\hat{v}$ & $\tilde{v}(95 \% \mathrm{CI})$ & $\hat{v}$ \\
\hline \hline 1 & 22.93 & 7 & 10.726 & 1.532 & $10.72( \pm 0.0034)$ & 1.53 \\
\hline 2 & 16.97 & 7 & 9.077 & 1.439 & $10.08( \pm 0.0029)$ & 1.44 \\
\hline 3 & 13.56 & 7 & 9.642 & 1.377 & $9.64( \pm 0.0026)$ & 1.38 \\
\hline 4 & 11.22 & 7 & 9.331 & 1.333 & $9.33( \pm 0.0018)$ & 1.33 \\
\hline 5 & 9.47 & 7 & 9.108 & 1.301 & $9.10( \pm 0.0020)$ & 1.30 \\
\hline 6 & 8.11 & 7 & 8.972 & 1.281 & $8.97( \pm 0.0020)$ & 1.28 \\
\hline 7 & 7 & 7 & 8.913 & 1.273 & $8.91( \pm 0.0020)$ & 1.27 \\
\hline
\end{tabular}

For Random Waypoint with hot-spots, we also use our custom simulator for the measurement of inter-contact times of a pair of mobile nodes, $A$ and $B$, moving in a square area of size $6000 \mathrm{~m} \times 6000 \mathrm{~m}$. A square region of width $500 \mathrm{~m}$ is designated as the hot-spot for $A$. The coordinate of the square hot-spot is set at $(1500,1500)$. A square region of the same size is placed at $(4500,4500)$ as a hot-spot for node $B$. To obtain the inter-contact time characteristics shown in our experimental results, for each mobile node the probability of choosing its hot-spot as its next waypoint is varied between $0.7-0.8$, and the pause time inside the hot-spot is set to $300 \mathrm{~s}$ and the pause time outside the hot-spot is set to $3 s$.

\subsection{Mobility Characteristics}

Below we discuss the simulation results for relative velocities and inter-contact times of mobile nodes. The relative velocity of mobile nodes is important in the calculation inter-contact times of mobile nodes. Along with the RWP factor $\rho$ discussed in Section 3, the relative velocity explains the differences in inter-contact times between Random Waypoint and Random Direction models.

\subsubsection{Relative Velocity of Mobile Nodes}

In the calculation of the relative speed of mobile nodes under Random Waypoint model, we assumed that the relative movement angle is uniformly distributed, as described in [1]. To see we measure the relative movement angle of two mobile nodes moving according to RWP model in square and circular regions.

Figure 2 shows the histograms of relative movement angles of nodes moving according to the RWP model in circular and square regions, respectively. We can see that the distribution is virtually uniform for both areas, confirming the proof given in [1].

In Section 3, we discussed that the relative movement speed of two mobile nodes not only depends on the average speed of a mobile node, but also on the selection of the

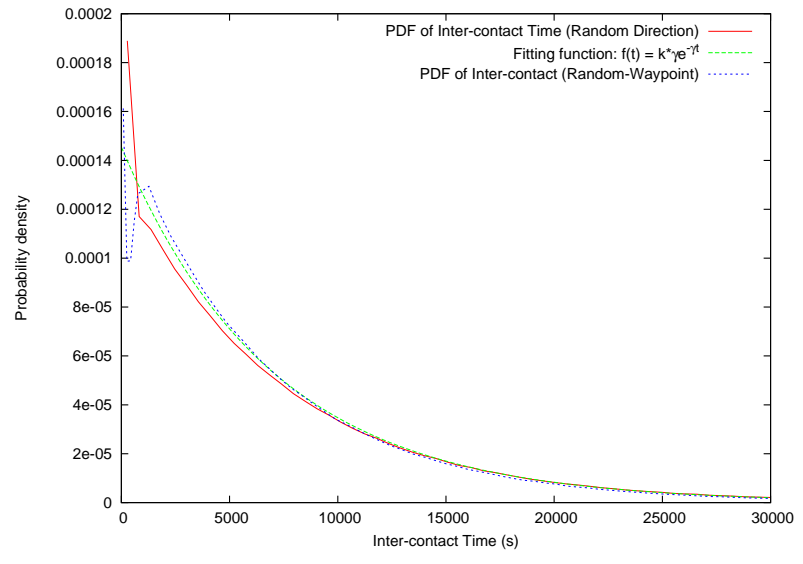

Figure 5. Inter-contact Rates of Random Waypoint and Random Direction Model Compared to Exponential Distribution

minimum speed, $v_{\min }$, and maximum speed $v_{\max }$.

Table 1 shows the effect of the distribution of $v_{\min }$ and $v_{\max }$ on relative speed, $\tilde{v}$. For this, we choose seven pairs of $v_{\min }$ and $v_{\max }$ that produce the same node average speed, $\bar{v}=7$. In this table, the $95 \%$ confidence interval for the average relative speed of mobile nodes. To see the effects, we define normalized speed $\hat{v}$ as $\hat{v}=\tilde{v} / \bar{v}$. We can see that $\hat{v}$ changes as the distribution of minimum and maximum speeds, and that empirical results confirms the results given in Equation (2).

\subsubsection{Inter-contact Times}

We first look at inter-contact times among mobile nodes. Figure 3(a) shows the histogram of inter-contact times under Random Waypoint model. To test the exponentiality of inter-contact times, we perform linear regression analysis on the Quantile-Quantile (Q-Q) plot of two data sets: recorded inter-contact times and randomly generated exponential variates with the same average as that of the experimental data. For linear regression, we consider three factors: coefficient of determination $\left(R^{2}\right)$, slope $a$, and intercept $b$. As shown in Figure 3(b), the recorded inter-contact times closely match the exponentially generated variates, with $R^{2}=0.999, a=0.995$, and $b=31.03$ (0.5\% of average). Similar results are obtained for Random Direction model as shown in Figure 4.

Although it has been shown that the inter-contact times can be very closely approximated as exponential under our experimental settings, a more detailed look show that inter-contact times differ from exponential distribution for smaller inter-contact times as depicted in Figure 5, where the number of bins used to get the histogram of inter-contact times is increased to 200, as opposed to 40 used in Figures 


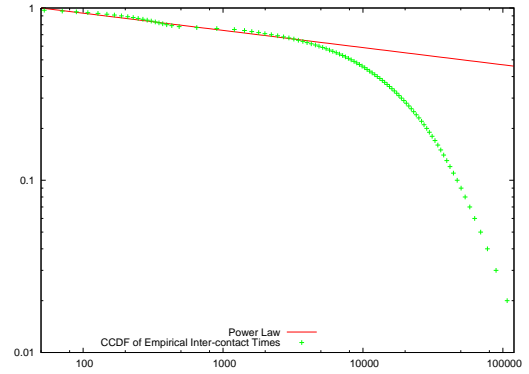

(a) Power Law

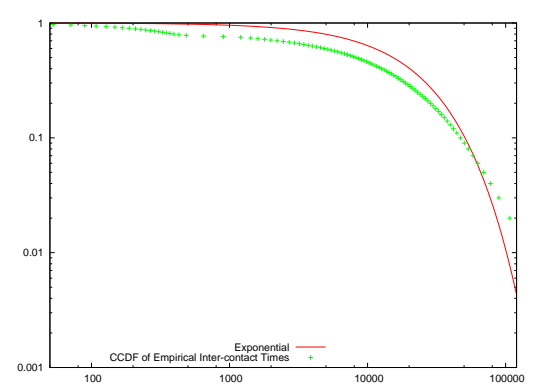

(b) Exponentia

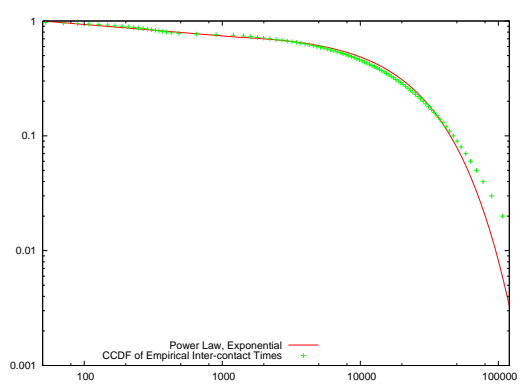

(c) Power Law + Exponential

Figure 6. Fitting the inter-contact rate using power law and exponential distribution

3(a) and 4(a). We can see that for inter-contact times less than 3000 seconds, the inter-contact time distributions of both Random Waypoint and Random Direction differ from exponential distribution in different ways. This shows that although exponentiality assumption holds for both mobility models, such differences should be considered for simulations that assume that the inter-contact times are strictly exponential, especially for small inter-contact times.

Figure 6 shows the approximations of inter-contact times under Random Waypoint with hot-spots. When the pause times are set exponentially both within and without the hotspots, we observe similar exponential behavior in intercontact times. However, when we set the pause time at hotspots according to power-law without changing the pause time outside of hotspots, we observe changes in intercontact times. As shown in Figures 6(a) and 6(b), neither power-law nor exponential distribution explains the distribution. If, however, power-law approximation is used up-to a time (until 1400 seconds in this case), and exponential afterwards, the approximations fit the experimental data fairly closely. This is a useful observation, as it conforms to the observations that are made in [14] for real-world mobility traces.

\section{Conclusions}

In this paper, we studied the properties of two commonly-used mobility models: Random Waypoint and Random Direction. We performed a detailed simulation study of inter-contact times under these models. We observe that the exponentiality assumption of inter-contact times generally hold under opportunistic network condition where radio range of nodes is much smaller compared to the radius of the system area. We also showed that the distributions of inter-contact times for smaller inter-contact times differ from exponential distribution under both mobility models in different ways. We also presented experimental results for extended Random Waypoint model with hotspots. We showed that the power-law head and exponen- tial tail of inter-contact times that are observed in real-world mobility traces can be produced under certain settings in this extended model.

\section{References}

[1] Muhammad Abdulla and Robert Simon. Characteristics of common mobility models for opportunistic networks. Crete, Greece, October 2007. ACM PM2HW2N'07.

[2] C. Bettstetter, H. Hartenstein, and X. Pérez-Costa. Stochastic properties of the random waypoint mobility model: Epoch length, direction distribution and cell change rate. In MSWIM '02. ACM Press, 2002.

[3] C. Bettstetter, G. Resta, and P. Santi. The node distribution of the random waypoint mobility model for wireless ad hoc networks. IEEE Trans. on Mobile Computing, 02(3):257-269, 2003.

[4] Christian Bettstetter. Mobility modeling in wireless networks: Categorization, smooth movement, and border effects. ACM Mobile Computing and Communications Review, 5(6):55-67, July 2001.

[5] Christian Bettstetter. Smooth is better than sharp: a random mobility model for simulation of wireless networks. In MSWIM '01, 2001.

[6] J. Le Boudec and M. Vojnovi. Perfect simulation and stationarity of a class of mobility models. INFOCOM, May 2005.

[7] A. Chaintreau, P. Hui, J. Crowcroft, C. Diot, R. Gass, and J. Scott. Impact of human mobility on the design of opportunistic forwarding algorithms. In $I N$ FOCOM, pages 1-13, April 2006.

[8] A. Jardosh et. al. Towards realistic mobility models for mobile ad hoc networks. In MobiCom '03, New York, NY, USA, 2003. ACM Press. 
[9] X. Hong et. al. A group mobility model for ad hoc wireless networks. In MSWiM '99, pages 53-60, New York, NY, USA, 1999. ACM Press.

[10] M. Garetto and E. Leonardi. Analysis of random mobility models with PDEs. In MobiHoc '06, pages 7384, 2006.

[11] R. Groenevelt, P. Nain, and G. Koole. The message delay in mobile ad hoc networks. In Performance, 2005.

[12] Sushant Jain, Kevin Fall, and Rabin Patra. Routing in a delay tolerant network. ACM SIGCOMM'04, August 2004.

[13] Evan P. C. Jones, Lily Li, and Paul A. S. Ward. Practical routing in delay-tolerant networks. In WDTN '05: Proceeding of the 2005 ACM SIGCOMM workshop on Delay-tolerant networking, pages 237-243. ACM, 2005 .

[14] T. Karagiannis, J.-Y. Le Boudec, and M. Vojnovic. Power law and exponential decay of inter contact times between mobile devices. Technical Report MSR-TR-2007, Microsoft Research, March 2007.

[15] Pasi Lassila. Spatial node distribution of the random waypoint mobility model with applications. IEEE Trans. on Mobile Computing, 5(6):680-694, 2006.

[16] P. Nain, D. Towsley, B. Liu, and Z. Liu. Properties of random direction models. In Proc. IEEE Infocom '05. ACM Press, March 2005.

[17] W. Navidi and T. Camp. Stationary distributions for the random waypoint mobility model. IEEE Transactions on Mobile Computing, 03(1):99-108, 2004.

[18] C. Perkins and P. Bhagwat. Routing over a multihop wireless network of mobile computers. Mobile Computing, 1996.

[19] C. Perkins and E. Royer. Ad hoc on-demand distance vector routing. Proc. of the 2nd IEEE Workshop on Mobile Comp. Sys. and Appl., 1999.

[20] R. Shah, S. Roy, S. Jain, and W. Brunette. Data mules: Modeling a three-tier architecture for sparse sensor networks. IEEE SNPA, 2003.

[21] T. Small and Z. J. Haas. Resource and performance tradeoffs in delay-tolerant wireless networks. In WDTN '05, pages 260-267, 2005.

[22] A. Spuropoulos, K. Psounis, and C. Raghavendra. Multi-copy routing in intermittently connected mobile networks. Technical Report CENG-2004-12, USC, 2004.
[23] T. Spyropoulos, K. Psounis, and C. Raghavendra. Single-copy routing in intermittently connected mobile networks. In Sensor and Ad Hoc Communications and Networks, pages 235-244. IEEE, Oct 2004.

[24] T. Spyropoulos, K. Psounis, and C. Raghavendra. Spray and wait: An efficient routing scheme for intermittently connected mobile networks. Philadelphia, PA, USA, August 2005. ACM SIGCOMM'05 Workshops.

[25] T. Spyropoulos, K. Psounis, and C. Raghavendra. Performance analysis of mobility-assisted routing. pages 49-60. ACM Mobihoc 2006, 2006.

[26] M. M. Bin Tariq, M. Ammar, and E. Zegura. Message ferry route design for sparse ad hoc networks with mobile nodes. In MobiHoc, 2006.

[27] J. Yoon, M. Liu, and B. Noble. Random waypoint considered harmful. INFOCOM, 2003.

[28] X. Zhang, G. Neglia, J. Kurose, and D. Towsley. Performance modeling of epidemic routing. Technical Report CMPSCI 2005-44, UMass, 2005.

[29] W. Zhao, M. Ammar, and E. Zegura. A message ferrying approach for data delivery in sparse mobile ad hoc networks. MobiHoc, May 2004.

\section{A Calculating the Value of $\rho$ for Circular Area}

To calculate the $\rho$ value of the circular region, we consider a unit disk of size $\pi$. From the polynomial approximations of the PDF of node distribution given by [15] for unit disk, we use the following:

$$
P(r)=\frac{3\left(1-r^{2}\right)\left(189-44 r^{2}-18 r^{4}\right)}{257 \pi}
$$

With this function, we calculate the value of $\rho$ as follows:

$$
\begin{aligned}
\rho & =A \iint_{\mathcal{A}} f^{2}(x, y) d x d y \\
& \approx \pi \int_{0}^{2 \pi} \int_{0}^{1} P^{2}(r) r d r d \phi \\
& =18 \pi^{2} \int_{0}^{1} \frac{r\left(1-r^{2}\right)^{2}\left(189-44 r^{2}-18 r^{4}\right)^{2}}{(257 \pi)^{2}} d r \\
& =\frac{3272289}{2311715} \\
& \approx 1.4155
\end{aligned}
$$

